\title{
Sodium-coupled Monocarboxylate Transporters in Normal Tissues and in Cancer
}

\author{
Vadivel Ganapathy, ${ }^{1,2}$ Muthusamy Thangaraju, ${ }^{1}$ Elangovan Gopal, ${ }^{1}$ Pamela M. Martin, ${ }^{1}$ \\ Shiro Itagaki, ${ }^{1}$ Seiji Miyauchi, ${ }^{1}$ and Puttur D. Prasad ${ }^{1}$
}

Received 23 January 2008; accepted 18 February 2008; published online 2 April 2008

\begin{abstract}
SLC5A8 and SLC5A12 are sodium-coupled monocarboxylate transporters (SMCTs), the former being a high-affinity type and the latter a low-affinity type. Both transport a variety of monocarboxylates in a $\mathrm{Na}^{+}$-coupled manner. They are expressed in the gastrointestinal tract, kidney, thyroid, brain, and retina. SLC5A8 is localized to the apical membrane of epithelial cells lining the intestinal tract and proximal tubule. In the brain and retina, its expression is restricted to neurons and the retinal pigment epithelium. The physiologic functions of SLC5A8 include absorption of short-chain fatty acids in the colon and small intestine, reabsorption of lactate and pyruvate in the kidney, and cellular uptake of lactate and ketone bodies in neurons. It also transports the B-complex vitamin nicotinate. SLC5A12 is also localized to the apical membrane of epithelial cells lining the intestinal tract and proximal tubule. In the brain and retina, its expression is restricted to astrocytes and Müller cells. SLC5A8 also functions as a tumor suppressor; its expression is silenced in tumors of colon, thyroid, stomach, kidney, and brain. The tumor-suppressive function is related to its ability to mediate concentrative uptake of butyrate, propionate, and pyruvate, all of which are inhibitors of histone deacetylases. SLC5A8 can also transport a variety of pharmacologically relevant monocarboxylates, including salicylates, benzoate, and $\gamma$-hydroxybutyrate. Non-steroidal anti-inflammatory drugs such as ibuprofen, ketoprofen, and fenoprofen, also interact with SLC5A8. These drugs are not transportable substrates for SLC5A8, but instead function as blockers of the transporter. Relatively less is known on the role of SLC5A12 in drug transport.
\end{abstract}

KEYWORDS: gamma-hydroxybutyrate; kidney/intestine; monocarboxylate drugs; SLC5A8/SLC5A12; tumor suppressor.

\section{INTRODUCTION}

Until recently, the only class of plasma membrane transporters which are known to mediate the uptake of monocarboxylates such as lactate, pyruvate, and short-chain fatty acids in mammalian cells was the $\mathrm{H}^{+}$-coupled monocarboxylate transporters (MCTs) $(1,2)$. MCTs belong to the solute carrier gene family SLC16 and mediate the cotransport of monocarboxylic substrates with $\mathrm{H}^{+}$with a $\mathrm{H}^{+}$: substrate stoichiometry of 1:1. Consequently, the transport process via MCTs is electroneutral. Recently, a second class of monocarboxylate transporters has been identified. There are two members in this class, and both of them mediate the cellular uptake of monocarboxylic substrates in a $\mathrm{Na}^{+}$coupled manner. This novel class of monocarboxylate transporters, known as sodium-coupled MCTs (SMCTs), plays an important role in the handling of a wide variety of endogenous monocarboxylates in various tissues. In addition, one member of this class functions as a tumor suppressor with

\footnotetext{
${ }^{1}$ Department of Biochemistry and Molecular Biology, Medical College of Georgia, Augusta, Georgia 30912, USA.

${ }^{2}$ To whom correspondence should be addressed. (e-mail: vganapat@ mcg.edu)
}

therapeutic potential in cancer treatment and also as a drug transporter with potential to modulate oral absorption and pharmacokinetics of various monocarboxylic drugs.

\section{MOLECULAR AND FUNCTIONAL IDENTIFICATION OF SODIUM-COUPLED MCTS}

In 2003, Li et al. (3) identified a transporter gene which functions as a tumor suppressor in human colon. This gene, $S L C 5 A 8$, belongs to the solute carrier gene family $S L C 5$. The gene is expressed in normal colon, but silenced in colon cancer and also in a variety of colon cancer cell lines. The silencing involves DNA methylation. Ectopic expression of the transporter in transporter-deficient colon cancer cell lines leads to suppression of colony-forming ability of these cells, thus establishing the tumor-suppressive function of the transporter. Even though the studies by $\mathrm{Li}$ et al. (3) established the role of this transporter as a tumor suppressor, the actual function of the transporter was not identified. Li et al. (3) called this transporter a "sodium transporter" because it belongs to the $S L C 5$ gene family which consists of $\mathrm{Na}^{+}-$ coupled transporters for a variety of substrates (Table I). The ability of SLC5A8 to transport $\mathrm{Na}^{+}$was demonstrated by these investigators in $X$. laevis oocytes following heterologous 
Table I. Members of the $S L C 5$ Gene Family

\begin{tabular}{ccl}
\hline HUGO nomenclature & Common name & \multicolumn{1}{c}{ Transport function } \\
\hline SLC5A1 & SGLT1 & $\begin{array}{l}\mathrm{Na}^{+} / \text {glucose or } \\
\mathrm{Na}^{+} / \text {galactose }\end{array}$ \\
SLC5A2 & SGLT2 & $\mathrm{Na}^{+} /$glucose \\
SLC5A3 & SMIT1 & $\mathrm{Na}^{+} /$myoinositol \\
SLC5A4 & SGLT3 & $\begin{array}{l}\mathrm{Glucose}^{+} \text {sensitive } \\
\mathrm{Na}^{+} \text {-channel }\end{array}$ \\
SLC5A5 & NIS & $\mathrm{Na}^{+} /$iodide \\
SLC5A6 & SMVT & $\mathrm{Na}^{+} /$biotin or \\
& & $\mathrm{Na}^{+} /$pantothenate \\
SLC5A7 & CHT1 & $\mathrm{Na}^{+} /$Cl $/$choline \\
SLC5A8 & SMCT1 & $\mathrm{Na}^{+} /$monocarboxylate \\
SLC5A9 & SMT & $\mathrm{Na}^{+} /$mannose \\
SLC5A10 & $?$ & $\mathrm{Unknown}^{2}$ \\
SLC5A11 & SMIT2 & $\mathrm{Na}^{+} /$myoinositol \\
SLC5A12 & SMCT2 & $\mathrm{Na}^{+} /$monocarboxylate \\
\hline
\end{tabular}

SGLT Sodium/glucose cotransporter, SMIT sodium/myoinositol cotransporter, NIS $\mathrm{Na}^{+}$/iodide symporter, SMVT sodium-coupled multivitamin transporter, CHT choline transporter, SMT sodium/ mannose cotransporter, $S M C T$ sodium-coupled monocarboxylate transporter, $H U G O$ human genome organization

expression of the transporter, but the substrate that was cotransported with $\mathrm{Na}^{+}$was never identified. We had cloned the same transporter a few years earlier from a kidney cDNA library, but were unable to establish its transport function. The findings by Li et al. (3) showed for the first time that the transporter is expressed not only in the kidney but also in the colon and that the function of the transporter is somehow related to tumor suppression. These new findings provided an important clue as to the actual transport function of SLC5A8. It has been known for several decades that fiber intake in the diet reduces the risk for colon cancer and that bacterial fermentation of fiber in the colonic lumen generates shortchain fatty acids which are likely to be responsible for this tumor-suppressive effect $(4,5)$. These bacterial metabolites are monocarboxylates which include acetate, propionate, and butyrate. Among these, butyrate is an inhibitor of histone deacetylases and is known to promote differentiation in normal colon epithelial cells but induce apoptosis selectively in tumor cells. Based on these findings, we hypothesized that SLC5A8 may function as a transporter for these short-chain fatty acids. If SLC5A8 functions as a $\mathrm{Na}^{+}$-coupled transporter for butyrate, it would provide a logical explanation for its expression in normal colon where cellular uptake of butyrate via the transporter might underlie the biologic actions of this bacterial metabolite in colon epithelial cells. It would also provide a molecular basis for the tumor-suppressive function of the transporter and a rationale why the expression of the transporter is silenced in cancer. Therefore, we tested whether SLC5A8 could function as a transporter for butyrate and other short-chain fatty acids. These studies led to the discovery that SLC5A8 is indeed a transporter for monocarboxylates and that the transport process is $\mathrm{Na}^{+}$dependent and electrogenic (6). Curiously, the ability of SLC5A8 to transport short-chain fatty acids does not explain why the transporter is expressed at high levels in the kidney because the concentrations of these monocarboxylates in circulation are very low. But, lactate and pyruvate, which are also monocarboxylates similar to short-chain fatty acids, are present in significant amounts in circulation and these metabolites are efficiently reabsorbed in the kidney with minimal urinary excretion. This suggests that renal tubular cells must possess an active reabsorption mechanism for these monocarboxylates. In fact, a $\mathrm{Na}^{+}$-coupled transport system for lactate and pyruvate has been identified at the functional level in brush-border membrane of renal tubular cells (7), but the transporter responsible for the function was not identified at the molecular level. Therefore, we hypothesized that SLC5A8 may be the transporter that is responsible for the renal reabsorption of lactate and pyruvate. Subsequent studies with lactate and pyruvate as substrates showed that SLC5A8 also functions as a $\mathrm{Na}^{+}$-coupled transporter for these monocarboxylates $(6,8)$. The transport process is electrogenic, suggesting that more than $1 \mathrm{Na}^{+}$is transported per transport cycle with a $\mathrm{Na}^{+} /$monocarboxylate substrate ratio of $\geq 2$. The affinity of the transporter for these monocarboxylates is fairly high, with Michaelis constant in the range of $200-400 \mu \mathrm{M}$ (Table II). The identity of SLC5A8 as a $\mathrm{Na}^{+}$-coupled and electrogenic transporter for short-chain fatty acids and lactate has been confirmed independently by two other groups of investigators $(9,10)$. Screening of additional monocarboxylates as potential substrates for SLC5A8 has revealed that nicotinate (a B-complex vitamin) and acetoacetate and $\beta$-hydroxybutyrate (also known as ketone bodies) also serve as substrates for the transporter $(11,12)$.

While screening a mouse kidney cDNA library which yielded SLC5A8 cDNA, we isolated a related cDNA from the same library which codes for a transporter protein highly homologous to SLC5A8. This transporter, known as SLC5A12, also belongs to the same gene family as does

Table II. Affinities for Various Monocarboxylates for Transport Via Human SLC5A8

\begin{tabular}{lc}
\hline \multicolumn{1}{c}{ Monocarboxylate } & $K_{\mathrm{m}}(\mu \mathrm{M})$ \\
\hline Acetate & $2,460 \pm 890^{\mathrm{a}}$ \\
Propionate & $127 \pm 14^{\mathrm{a}}, 128 \pm 4^{\mathrm{b}}, 162 \pm 26^{\mathrm{c}}$ \\
Butyrate & $72 \pm 8^{\mathrm{b}}, 81 \pm 17^{\mathrm{a}}$ \\
L-Lactate & $159 \pm 24^{\mathrm{b}}, 184 \pm 8^{\mathrm{d}}, 235 \pm 24^{\mathrm{a}}$ \\
D-Lactate & $742 \pm 330^{\mathrm{a}}, 1,088 \pm 68^{\mathrm{d}}$ \\
Pyruvate & $387 \pm 43^{\mathrm{d}}$ \\
Nicotinate & $230 \pm 16^{\mathrm{e}}, 390 \pm 36^{\mathrm{c}}$ \\
Acetoacetate & $213 \pm 39^{\mathrm{d}}$ \\
$\beta$-D-Hydroxybutyrate & $1,442 \pm 124^{\mathrm{d}}$ \\
$\beta$-L-Hydroxybutyrate & $2,327 \pm 169^{\mathrm{d}}$ \\
$\gamma$-Hydroxybutyrate & $1,620 \pm 86^{\mathrm{f}}$ \\
$\alpha$-Ketoisocaproate & $209 \pm 27^{\mathrm{d}}$ \\
Benzoate & $1,100 \pm 200^{\mathrm{e}}$ \\
Salicylate & $1,500 \pm 100^{\mathrm{e}}$ \\
5-Aminosalicylate & $6,500 \pm 1,000^{\mathrm{e}}$ \\
\hline
\end{tabular}

${ }^{a}$ Values are from (6).

${ }^{b}$ Values are from (9).

${ }^{c}$ Values are from (10).

${ }^{d}$ Values are from (12).

${ }^{e}$ Values are from (16).

${ }^{f}$ Values are from present study.

Values were determined using substrate-induced currents as a measure of transport in $X$. laevis oocytes expression system, following heterologous expression of human SLC5A8. Data are from (6), (9), (10), (12), and (16). 
SLC5A8, and is expressed primarily in the kidney and intestinal tract, and to a lesser extent in the brain and retina. We have now established the transport function of SLC5A12 $(13,14)$. It also mediates $\mathrm{Na}^{+}$-coupled transport of monocarboxylates, but the transport process seems to be electroneutral with a $\mathrm{Na}^{+}$:monocarboxylate stoichiometry of $1: 1$. The substrate selectivity of SLC5A12 is similar to that of SLC5A8, but SLC5A12 is a low-affinity transporter while SLC5A8 is a high-affinity transporter.

\section{EXPRESSION PATTERN OF SLC5A8 AND SLC5A12 IN THE INTESTINAL TRACT AND KIDNEY}

SLC5A8 is expressed differentially in the lumen-facing apical membrane of colonic and intestinal epithelial cells $(15,16)$. This localization is appropriate to its postulated physiologic role in the cellular uptake of short-chain fatty acids which are generated in the colonic/intestinal lumen by bacterial fermentation of dietary fiber. The expression pattern of SLC5A12 in the intestinal tract has not been studied. In the kidney, SLC5A8 is expressed in the apical membrane of tubular epithelial cells and the expression is restricted principally to the straight portion of the proximal tubule (S3 segment) (14). In contrast, SLC5A12 is expressed also in the apical membrane of tubular epithelial cells but its expression is evident all along the proximal tubule (S1, S2, and S3 segments) (14). This makes sense in terms of the proposed physiologic role of these transporters in the renal reabsorption of lactate. Plasma levels of lactate are $1-1.5 \mathrm{mM}$. Therefore, the concentration of lactate in the tubular filtrate is expected to be in millimolar range in the initial portions of the proximal tubule. A low-affinity transporter such as SLC5A12 is appropriate to mediate effective reabsorption of lactate at these concentrations. Accordingly, this transporter is expressed at high levels in epithelial cells lining the initial portions of the proximal tubule. As lactate reabsorption continues via SLC5A12 along the proximal tubule, the concentration is expected to fall as the fluid moves along the tubule, and a transporter with relatively higher affinity for lactate is appropriate to mediate lactate reabsorption under these conditions. SLC5A8 satisfies this need. Accordingly, this high-affinity transporter is expressed primarily in the late portions of the proximal tubule. Thus, the segmental localization of these two transporters is suitable for effective reabsorption of lactate in the kidney. The obligatory role of these transporters in lactate reabsorption in the kidney has recently been confirmed using a transgenic mouse model (c/ebp $\delta$ null mouse) (17). $\mathrm{C} / \mathrm{EBP} \delta$ is a member of the CCAAT/enhancer binding protein (C/EBP) family (18). $c / e b p \delta^{-/}$mice are viable, but exhibits defects in adipocyte differentiation (19), learning and memory (20), and mammary gland involution $(21,22)$. We recently found that the expression of SLC5A8 as well as SLC5A12 in the kidney is markedly decreased in $c / e b p \delta^{-/-}$mice (17). The expression of various other transporters is not affected. The decreased expression of SLC5A8 and SLC5A12 leads to massive urinary excretion of lactate in these mice with concomitant decrease in plasma levels of lactate. This mouse model also proved to be useful to establish a functional link between lactate reabsorption and urate reabsorption in the kidney. In normal kidney, reabsorption of urate is mediated by URAT1, an anion exchanger with lactate being the principal exchangeable anion (23). Even though there is no change in the expression levels of URAT1 and many other transporters in $c / e b p \delta^{-/}$mice, urinary excretion of urate is increased significantly (17). This indicates that effective cellular accumulation of lactate in proximal tubular cells via SLC5A8/ SLC5A12 is necessary for optimal reabsorption of urate in normal kidney. The defective reabsorption of lactate in $c / e b p \delta^{-/}$ mouse kidneys as a consequence of decreased expression of SLC5A8 and SLC5A12 leads to an impairment in the renal reabsorption of urate.

\section{EXPRESSION OF SLC5A8 AND SLC5A12 IN THE BRAIN AND RETINA AND THEIR POTENTIAL BIOLOGIC ROLE}

The findings that SLC5A8 is able to transport lactate and ketone bodies ( $\beta$-hydroxybutyrate and acetoacetate) (12) prompted us to examine the expression of the transporter in the brain. It is well known that increased brain activity is associated with increased utilization of glucose, but the uptake and metabolism of glucose is enhanced not in activated neurons but in astrocytes. Glutamate, the major neurotransmitter in the brain, links neuronal activation to astrocyte metabolism to enhance glucose breakdown via glycolysis, leading to generation and release of lactate from the astrocytes (24). Activated neurons then take up lactate to replenish their energy stores. Similarly, when glucose is not available as in prolonged starvation or is not metabolically usable as in untreated diabetes, neurons in the brain rely on ketone bodies as the energy source. These ketone bodies are generated by the liver and also by the astrocytes via $\beta$-oxidation of fatty acids. Thus, there is a close functional relationship between neurons and astrocytes in terms of energy metabolism. Astrocytes provide metabolic support to activated neurons by generating lactate and ketone bodies as the immediate energy source for neurons. This model of neuroenergetics necessitates expression of efficient transporters in neurons for uptake of lactate and ketone bodies. MCTs are known to play a role in this process (25). There is a differential expression of MCT isoforms in neurons versus astrocytes, with MCT2 principally in neurons and MCT1 and MCT4 in astrocytes. However, MCTs are not very effective in concentrative uptake of lactate and ketone bodies because of their energetics (coupling with transmembrane $\mathrm{H}^{+}$gradient and electroneutral nature of the transport process). In addition, MCTs exhibit low affinity for their substrates, the values for Michaelis constant generally being in millimolar range. Therefore, we asked if SLC5A8, a highly concentrative transporter for lactate and ketone bodies because of its coupling to transmembrane $\mathrm{Na}^{+}$gradient and the electrogenic nature of the transport process, is expressed in neurons. Our studies have shown that it indeed is the case (12). The expression pattern of SLC5A8 in the brain overlaps with that of MCT2 (a neuron-specific MCT), NeuN (a neuronal cell body marker) and MAP2 (a neuronal axonal marker). There is no overlap between SLC5A8 expression pattern and that of MCT1 (an astrocyte-specific MCT) and GFAP (a glial cell marker). These studies show that the high-affinity sodiumcoupled MCT SLC5A8 is expressed specifically in neurons in the brain, suggesting that the transporter might play an important role in the neuronal uptake of lactate and ketone bodies and thus in the maintenance of energy status of the 
neurons. In contrast, the low-affinity sodium-coupled MCT SLC5A12 is expressed specifically in astrocytes (26). The physiologic role of this transporter in astrocytes remains to be established. A similar expression pattern of SLC5A8 and SLC5A12 is also seen in the retina (26). SLC5A8 is expressed primarily in neurons and in the retinal pigment epithelium whereas SLC5A12 is expressed almost exclusively in Müller cells, the glial cells of the retina. Despite the differential expression of the isoforms of MCTs and SMCTs in neurons versus astrocytes and the in vitro evidence for their potential physiologic functions, the relevance of these transporters to the maintenance of energy status of the brain in vivo remains to be established.

\section{SLC5A8 AS A TUMOR SUPPRESSOR IN THE COLON: THE BUTYRATE AND HDAC CONNECTION}

The studies by Li et al. (3) have unequivocally shown that SLC5A8 functions as a tumor suppressor in the colon, but the molecular mechanism underlying its tumor-suppressive function was not known. We hypothesized that the ability of SLC5A8 to mediate concentrative uptake of butyrate, an inhibitor of histone deacetylases (HDACs), from the colonic lumen into colon epithelial cells might underlie the tumorsuppressive function of the transporter. To test this hypothesis, we screened several colon cell lines for the expression of the transporter. Unpublished data from our laboratory have shown that non-malignant colon cell lines express the transporter whereas malignant cell lines do not. Exposure of nonmalignant cell lines to butyrate does not induce apoptosis even though these cell lines express the transporter, indicating that butyrate entry into cells is not detrimental to non-malignant cells. Exposure of malignant cells to butyrate also does not induce apoptosis. This is predictable because of the absence of SLC5A8 and consequently the absence of concentrative uptake of butyrate in these cells. When SLC5A8 is expressed ectopically in malignant cells, there is no effect as long as the culture medium does not contain butyrate, showing that the expression of the transporter by itself alone is insufficient to induce apoptosis. In other words, the transporter protein per se without its transport function is not a tumor suppressor. But, when these cells are cultured in the presence of butyrate, cells undergo massive apoptosis (unpublished data). These studies show that the ability of SLC5A8 to transport butyrate actively into cells is most likely responsible for its tumor-suppressive function in the colon. The relevance of HDAC inhibition to apoptosis in these cells is demonstrable by examining the acetylation status of histones. Several studies have shown that the bacterial fermentation product butyrate causes HDAC inhibition and suppresses cell proliferation (27-29). SLC5A8 provides a molecular link between the commensal bacteria in the colonic lumen and the host because the transporter is essential to mediate the beneficial effects of the bacterial metabolite butyrate in the colon $(30,31)$.

\section{SLC5A8 AS A TUMOR SUPPRESSOR IN NON-COLONIC TISSUES: MECHANISM OF TUMOR SUPPRESSION}

The findings that butyrate functions as a tumor suppressor via SLC5A8 are physiologically relevant only to the colon because this tissue is exposed to butyrate from the luminal side as a result of bacterial fermentation of dietary fiber. The expression of the transporter in the lumen-facing apical membrane of colonic epithelial cells agrees with this physiologic role. Interestingly, the expression of the transporter has been shown to be silenced also in cancer of a variety of noncolonic tissues, including thyroid, stomach, brain, breast, pancreas, and kidney $(30,31)$. But, unlike the colon, these tissues are not exposed to butyrate to any significant extent under physiologic conditions. This raises the question as to why cancer progression in these non-colonic tissues is associated with silencing of the transporter. In studies by $\mathrm{Li}$ et al. (3), ectopic expression of SLC5A8 in malignant colon cells led to growth arrest, but there was no evidence of addition of butyrate to the culture medium in these experiments. This suggested to us that there must be a SLC5A8 substrate in the culture medium which behaves similar to butyrate as an HDAC inhibitor, and that this substrate must also be present in circulation. This would explain the observed induction of apoptosis in colon cancer cell lines even in the absence of butyrate upon ectopic expression of the transporter and also the silencing of the transporter in cancer of non-colonic tissues. To identify this 'mystery' substrate of SLC5A8, we decided to generate a stable cancer cell line expressing the transporter ectopically with the goal of screening for the compounds in the cell culture medium for their ability to induce apoptosis. We selected the breast cancer cell line MCF7 for this purpose. We have shown that breast cancer is also associated with the silencing of $S L C 5 A 8$ and that MCF7 cell line, being a malignant cell, does not express the transporter (32). Therefore, a MCF7 cell line engineered to express SLC5A8 stably would be ideal for the screening. Surprisingly, we were unable to generate such a stable cell line. In the regular medium generally recommended for the culture of MCF7 cells, ectopic expression of SLC5A8 invariably led to apoptosis, suggesting that the culture medium used in these studies also contained the 'mystery' substrate. We suspected that pyruvate could be this substrate because the culture medium for MCF7 cells is normally supplemented with $1 \mathrm{mM}$ pyruvate. SLC5A8 transports pyruvate with high affinity, but there was no indication in the literature for possible tumor-suppressive ability of this ubiquitous metabolite. Surprisingly, when we used the culture medium without supplementation with pyruvate, we were able to generate stable MCF7 cell lines expressing the transporter. These studies showed for the first time that pyruvate induces apoptosis in cancer cells in a SLC5A8dependent manner (32). Supplementation of the culture medium with pyruvate does not do any harm to MCF7 cells because of the lack of expression of SLC5A8; but ectopic expression of the transporter in these cells leads to apoptosis when cultured in the presence of pyruvate-supplemented culture medium. It was clear now that SLC5A8-mediated concentrative entry of pyruvate was able to induce apoptosis in cancer cells, but the mechanism remained to be established. Subsequent studies revealed that pyruvate is almost as potent as butyrate in its ability to inhibit HDACs (32). The ability of pyruvate to induce apoptosis in tumor cells via SLC5A8 provides a rationale for the silencing of the transporter in cancer of non-colonic tissues. These findings are also relevant to cancer-specific cellular metabolism. It has 

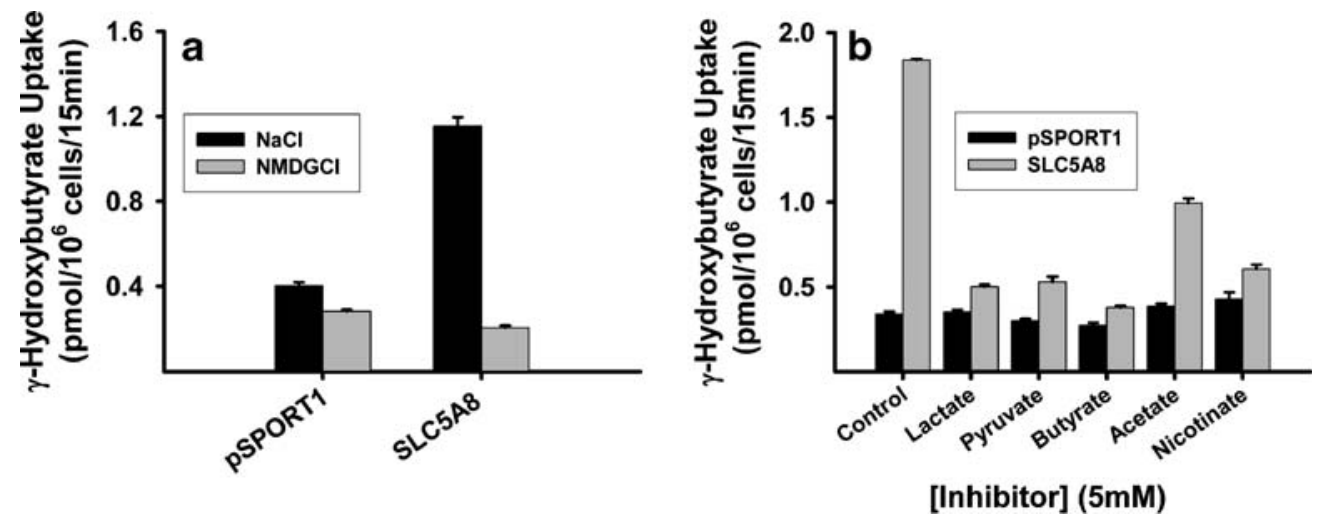

Fig. 1. Transport of $\gamma$-Hydroxybutyrate Via Human SLC5A8 in a Mammalian Cell Expression System. Human SLC5A8 Was Expressed Heterologously in Human Retinal Pigment Epithelial Cell Lines HRPE by cDNA Transfection. Cells Transfected with pSPORT1 Vector Served as the Control. a Uptake of $\left[{ }^{3} \mathrm{H}\right]-\gamma-$ Hydroxybutyrate $(25 \mathrm{nM})$ Was Measured in These Cells for $15 \mathrm{~min}$ in the Presence ( $\mathrm{NaCl} \mathrm{Buffer}$ ) or Absence ( $N$-methyl-D-glucamine Chloride Buffer) of $\mathrm{Na}^{+}$. b Uptake of $\left[{ }^{3} \mathrm{H}\right]-\gamma$-Hydroxybutyrate $(25 \mathrm{nM})$ Was Measured in a NaCl Buffer in Vector-transfected Cells and SLC5A8 cDNA-transfected Cells in the Presence or Absence of Unlabeled Monocarboxylate Substrates $(5 \mathrm{mM})$ of the Transporter

been known for decades that tumor cells upregulate glycolysis but silence mitochondrial oxidation $(33,34)$. The primary end product of cancer cell metabolism is lactate. Upregulation of glycolysis in the absence of mitochondrial oxidation leads to an increase in intracellular levels of pyruvate, but cancer cells avoid accumulation of this metabolite by effectively converting it to lactate. Interestingly, pyruvate is an inhibitor of HDACs while lactate is not (32). Thus, accumulation of pyruvate in cancer cells is not conducive to cell proliferation because of the ability of this metabolite to induce apoptosis via HDAC inhibition. In contrast, accumulation of lactate is not detrimental to cancer cells. Thus, cancer cells meet their energy demand by enhancing glycolysis even in the absence of mitochondrial oxidation, and prevent accumulation of pyruvate to avoid apoptosis by effectively converting pyruvate into lactate.

\section{RELEVANCE OF SLC5A8 TO DRUG TRANSPORT}

The expression of SLC5A8 in the lumen-facing apical membrane of epithelial cells lining the small intestine, colon, and the renal proximal tubule indicates that the transporter may influence oral bioavailability and renal reabsorption of monocarboxylate drugs. Potential substrates of SLC5A8 with pharmacological relevance include salicylates, benzoate, and non-steroidal anti-inflammatory drugs. Therefore, we examined the interaction of these monocarboxylates with SLC5A8 $(16,35)$. These studies have shown that benzoate, salicylate, and 5-aminosalicylate are transported via SLC5A8. $p$-Aminobenzoate is not recognized by the transporter. The transport of benzoate, salicylate, and 5-aminosalicylate via SLC5A8 is $\mathrm{Na}^{+}$-dependent and electrogenic, involving participation of at
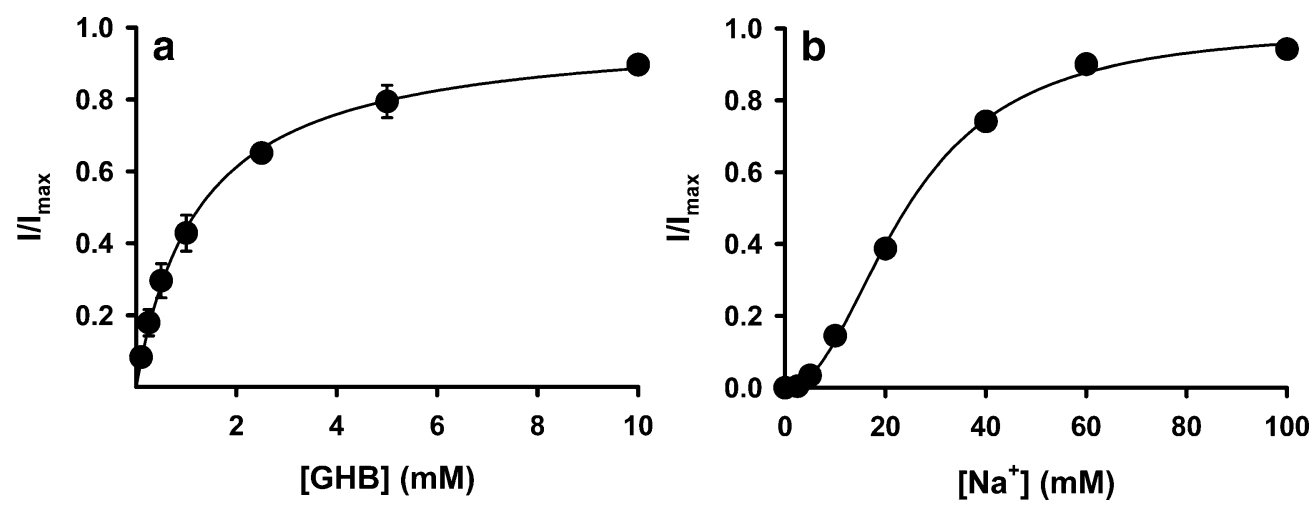

Fig. 2. Transport of $\gamma$-Hydroxybutyrate Via Human SLC5A8 in the X. laevis Oocyte Expression System. Human SLC5A8 Was Expressed Heterologously in $X$. laevis Oocytes by cRNA Injection. Water-injected Oocytes Served as the Control. Transport of $\gamma$-Hydroxybutryate via SLC5A8 was Monitored Electrophysiologically using the Two-microelectrode Voltage-clamp Technique, with Membrane Potential Clamped at $-50 \mathrm{mV}$. $\gamma$-Hydroxybutyrate (GHB) Induced Inward Currents in SLC5A8-expressing Oocytes. In Contrast, There Were No Currents in Water-injected Oocytes Under Similar Conditions. a Saturation Kinetics of GHB-induced Currents. b Na ${ }^{+}$-activation Kinetics of GHB-induced Currents. Since the Expression Levels Varied from Oocyte to Oocyte, the Maximal Current Induced in Each Oocyte Was Taken as 1, and the Currents Induced in the Same Oocyte at Different Experimental Conditions Were Expressed as a Fraction of this Maximal Current. The Michaelis Constant Was Calculated by Fitting the Experimental Data from Saturation Kinetics to Michaelis-Menten Equation Describing a Single Saturable System. The Hill Coefficient Was Calculated by Fitting the Experimental Data from $\mathrm{Na}^{+}$-activation Kinetics to Hill Equation 
least two $\mathrm{Na}^{+}$in the transport process. The Michaelis constant for these drugs is in the range of 1-7 mM. In contrast to these drugs, non-steroidal anti-inflammatory drugs such as ibuprofen, ketoprofen, and fenoprofen interact with the transporter but do not serve as transportable substrates (35). Ibuprofen is able to block the transport function of SLC5A8 by competing with transportable substrates such as lactate, nicotinate, and short-chain fatty acids, but is not transported. This is evident from the ability of ibuprofen to block the SLC5A8-mediated currents induced by transportable substrates and from the lack of transportermediated currents in the presence of ibuprofen itself. Thus, ibuprofen functions as a blocker of SLC5A8 transport function. These findings have pharmacologic and therapeutic significance. 5-Aminosalicylate is a drug used in the treatment of ulcerative colitis; therefore the intended target site for this drug is the colon. Preparations of this drug are designed either by specific formulation or in the form of prodrug such that 5-aminosalicylate is released primarily in the colonic lumen. Since the molecular target for this drug (cyclooxygenases) is located in the cytoplasm, the drug has to enter the colonic epithelial cells to elicit its pharmacologic effect. The expression of SLC5A8 in the apical membrane of these cells suggests that the transporter may be involved in facilitating the cellular entry of this drug. The findings that certain drugs such as ibuprofen function as blockers of this transporter raise the possibility for drug-drug interaction at the level of cellular uptake and oral bioavailability. A similar phenomenon is expected to occur in the kidney in terms of reabsorption of monocarboxylate drugs, potentially influencing their pharmacokinetics.

Since butyrate and $\beta$-hydroxybutyrate are transportable substrates for SLC5A8, we were curious to find out if $\gamma$ hydroxybutyrate serves as a transportable substrate for this transporter. $\gamma$-Hydroxybutyrate is an endogenous neuromodulator with significant psychotropic effects. It is also a drug of abuse. Our studies have shown that $\gamma$-hydroxybutyrate is transported via SLC5A8 in a $\mathrm{Na}^{+}$-dependent manner (Fig. 1a) and that this transport is inhibited competitively by other substrates of the transporter (Fig. 1b). We could also demonstrate SLC5A8-mediated transport of this drug by monitoring inward currents induced in $X$. laevis oocytes following heterologous expression of the transporter. The currents induced by $\gamma$-hydroxybutyrate are saturable with a Michaelis constant of $\sim 2 \mathrm{mM}$ (Fig. 2a). Analysis of $\mathrm{Na}^{+}-$ activation kinetics of the transport process showed a sigmoidal relationship between drug-induced currents and $\mathrm{Na}^{+}$concentration with a Hill coefficient of 2 (Fig. 2b). These data show that SLC5A8 offers a $\mathrm{Na}^{+}$-coupled electrogenic transport system for $\gamma$-hydroxybutyrate, suggesting that the transporter may play a role in the oral bioavailability and renal reabsorption of this drug. Table II summarizes the Michaelis constants $\left(K_{\mathrm{m}}\right)$ for various monocarboxylates for transport via human SLC5A8.

\section{SINGLE NUCLEOTIDE POLYMORPHISMS IN SLC5A8 AND THEIR INFLUENCE ON TRANSPORT FUNCTION}

A recent search of GenBank database indicates the existence of several single nucleotide polymorphisms in the gene coding for SLC5A8. While most of these SNPs are either located in introns or result in synonymous codons with no change in amino acid sequence, there are a few SNPs which result in amino acid substitutions. Many of these substitutions are conservative and therefore are unlikely to produce dramatic effects on transport function. One SNP, however, causes substitution of Val for Phe at position 251, which may exert significant influence on transport function because of the differences in the chemical structure (aliphatic versus aromatic) and bulkiness of the amino acids involved in the substitution. Therefore, we examined the transport function of the V251 variant and compared it with the transport function of the wild type transporter (F251). The V251 variant had significantly less transport activity compared to the wild type transporter (unpublished data). These data may have biologic relevance because of the tumorsuppressive function of the transporter. The SNP causing the substitution of Val for Phe may have significantly less tumorsuppressive effect. These findings may also have pharmacologic and therapeutic relevance because of the potential participation of the transporter in intestinal/colonic absorption and renal reabsorption of a variety of monocarboxylate drugs.

\section{CONCLUSIONS}

There are two sodium-coupled MCTs in mammalian cells: SLC5A8 and SLC5A12. SLC5A8 is a $\mathrm{Na}^{+}$-coupled, electrogenic, high-affinity transporter for short-chain fatty acids, lactate, pyruvate, nicotinate, and ketone bodies. It is expressed in the apical membrane of intestinal/colonic epithelial cells and renal proximal tubular cells, and in neurons in the brain and retina. It plays an essential role in the absorption of short-chain fatty acids and the B-complex vitamin nicotinate in the intestine and colon, in the reabsorption of lactate and other monocarboxylate substrates in the kidney, and in the active uptake of lactate and ketone bodies in neurons. This transporter also functions as a tumor suppressor. The ability of SLC5A8 to mediate concentrative accumulation of the histone deacetylase inhibitors butyrate and pyruvate underlies the tumorsuppressive function of this transporter in colonic and noncolonic tissues. SLC5A8 is also a $\mathrm{Na}^{+}$-coupled, electrogenic transporter for various monocarboxylate drugs such as benzoate, salicylates, and $\gamma$-hydroxybutyrate. SLC5A12 is also a $\mathrm{Na}^{+}$-coupled transporter for monocarboxylates, but its substrate affinity is much lower than that of SLC5A8, and its transport function is most likely electroneutral. Due to its low affinity, this transporter is most probably responsible for the bulk of lactate absorption in the kidney. It is expressed in the small intestine but not in the colon. In the brain and retina, SLC5A12 is expressed exclusively in astrocytes and glia. Little is known on the role of this transporter in drug transport.

\section{REFERENCES}

1. B. E. Enerson, and L. R. Drewes. Molecular features, regulation, and function of monocarboxylate transporters: implications for drug delivery. J. Pharm. Sci. 92:1531-1544 (2003).

2. A. P. Halestrap, and D. Meredith. The SLC16 gene family-from monocarboxylate transporters (MCTs) to aromatic amino acid 
transporters and beyond. Pflugers Arch. Eur. J. Physiol. 447:619628 (2004).

3. H. Li, L. Myeroff, D. Smiraglia, et al. SLC5A8, a sodium transporter, is a tumor suppressor gene silenced by methylation in human colon aberrant crypt foci and cancers. Proc. Natl. Acad. Sci. U. S. A. 100:8412-8417 (2003).

4. D. L. Topping, and P. M. Clifton. Short-chain fatty acids and human colonic function: roles of resistant starch and nonstarch polysaccharides. Physiol. Rev. 81:1031-1064 (2001).

5. Y. I. Kim. AGA technical review: impact of dietary fiber on colon cancer occurrence. Gastroenterology 118:1235-1257 (2000).

6. S. Miyauchi, E. Gopal, Y. J. Fei, and V. Ganapathy. Functional identification of SLC5A8, a tumor suppressor down-regulated in colon cancer, as a $\mathrm{Na}^{+}$-coupled transporter for short-chain fatty acids. J. Biol. Chem. 279:13293-13296 (2004).

7. M. Barac-Nieto, H. Murer, and R. Kinne. Lactate-sodium cotransport in rat renal brush border membranes. Am. J. Physiol. 239:F496-F506 (1980).

8. E. Gopal, Y. J. Fei, M. Sugawara, et al. Expression of slc5a8 in kidney and its role in $\mathrm{Na}^{+}$-coupled transport of lactate. J. Biol. Chem. 279:44522-44532 (2004).

9. M. J. Coady, M. H. Chang, F. M. Charron, et al. The human tumour suppressor gene SLC5A8 expresses a $\mathrm{Na}^{+}$-monocarboxylate cotransporter. J. Physiol. 557:719-731 (2004).

10. V. Paroder, S. R. Spencer, M. Paroder, et al. $\mathrm{Na}^{+} /$monocarboxylate transport (SMCT) protein expression correlates with survival in colon cancer: molecular characterization of SMCT. Proc. Natl. Acad. Sci. U. S. A. 103:7270-7275 (2006).

11. E. Gopal, Y. J. Fei, S. Miyauchi, L. Zhuang, P. D. Prasad, and V. Ganapathy. Sodium-coupled and electrogenic transport of Bcomplex vitamin nicotinic acid by slc5a8, a member of the $\mathrm{Na}^{+} /$ glucose cotransporter gene family. Biochem. J. 388:309-316 (2005).

12. P. M. Martin, E. Gopal, S. Ananth, et al. Identity of SMCT1 (SLC5A8) as a neuron-specific $\mathrm{Na}^{+}$-coupled transporter for active uptake of L-lactate and ketone bodies in the brain. J. Neurochem. 98:279-288 (2006).

13. S. R. Srinivas, E. Gopal, L. Zhuang, et al. Cloning and functional identification of slc5a12 as a sodium-coupled low-affinity transporter for monocarboxylates (SMCT2). Biochem. J. 392:655-664 (2005).

14. E. Gopal, N. S. Umapathy, P. M. Martin, et al. Cloning and functional characterization of human SMCT2 (SLC5A12) and expression pattern of the transporter in kidney. Biochim. Biophys. Acta. 1768:2690-2697 (2007).

15. T. Iwanaga, K. Takebe, I. Kato, S. Karaki, and A. Kuwahara. Cellular expression of monocarboxylate transporters (MCT) in the digestive tract of the mouse, rat, and humans, with special reference to slc5a8. Biomed. Res. 27:243-254 (2006).

16. E. Gopal, S. Miyauchi, P. M. Martin, S. Ananth, P. Roon, S. B. Smith, and V. Ganapathy. Transport of nicotinate and structurally related compounds by human SMCT1 (SLC5A8) and its relevance to drug transport in the mammalian intestinal tract. Pharm. Res. 24:575-584 (2007).

17. M. Thangaraju, S. Ananth, P. M. Martin, et al. c/ebpd null mouse as a model for the double-knockout of slc5a 8 and slc5a12 in kidney. J. Biol. Chem. 281:26769-26773 (2006).
18. P. F. Johnson. Molecular stop signs: regulation of cell-cycle arrest by C/EBP transcription factors. J. Cell Sci. 118:2545-2555 (2005).

19. T. Tanaka, N. Yoshida, T. Kishimoto, et al. Defective adipocyte differentiation in mice lacking the C/EBPbeta and/or C/EBPdelta gene. EMBO J. 16:7432-7443 (1997).

20. E. Sterneck, R. Paylor, V. Jackson-Lewis, et al. Selectively enhanced contextual fear conditioning in mice lacking the transcriptional regulator CCAAT/enhancer binding protein delta. Proc. Natl. Acad. Sci. U. S. A. 95:10908-10913 (1998).

21. A. P. Gigliotti, P. F. Johnson, E. Sterneck, et al. Nulliparous CCAAT/enhancer binding proteindelta (C/EBPdelta) knockout mice exhibit mammary gland ductal hyperlasia. Exp. Biol. Med. (Maywood). 228:278-285 (2003).

22. M. Thangaraju, M. Rudelius, B. Bierie, et al. C/EBPdelta is a crucial regulator of pro-apoptotic gene expression during mammary gland involution. Development. (Cambridge). 132:46754685 (2005).

23. N. Anzai, Y. Kanai, and H. Endou. New insights into renal transport of urate. Curr. Opin. Rheumatol. 19:151-157 (2007).

24. L. Pellerin. How astrocytes feed hungry neurons. Mol. Neurobiol. 32:59-72 (2005).

25. K. Pierre, and L. Pellerin. Monocarboxylate transporters in the central nervous system: distribution, regulation and function. J. Neurochem. 94:1-14 (2005).

26. P. M. Martin, Y. Dun, B. Mysona, S. Ananth, P. Roon, S. B. Smith, and V. Ganapathy. Expression of the sodium-coupled monocarboxylate transporters SMCT1 (slc5a8) and SMCT2 (slc5a12) in retina. Invest. Ophthalmol. Vis. Sci. 48:3356-3363 (2007).

27. J. S. Chen, D. V. Faller, and R. A. Spanjaard. Short-chain fatty acid inhibitors of histone deacetylases: promising anticancer therapeutics? Curr. Cancer Drug Targets. 3:219-236 (2003).

28. J. R. Davie. Inhibition of histone deacetylase activity by butyrate. J. Nutr. 133(Suppl. 7):2485S-2493S (2003).

29. M. C. Myzak, and R. H. Dashwood. Histone deacetylases as targets for dietary cancer preventive agents: lessons learned with butyrate, diallyl disulfide, and sulforaphane. Curr. Drug Targets. 7:443-452 (2006).

30. V. Ganapathy, E. Gopal, S. Miyauchi, and P. D. Prasad. Biological functions of SLC5A8, a candidate tumor suppressor. Biochem. Soc. Trans. 33:237-240 (2005).

31. N. Gupta, P. M. Martin, P. D. Prasad, and V. Ganapathy. SLC5A8 (SMCT1)-mediated transport of butyrate forms the basis for the tumor suppressive function of the transporter. Life Sci. 78:2419-2425 (2006)

32. M. Thangaraju, E. Gopal, P. M. Martin, et al. SLC5A8 triggers tumor cell apoptosis through pyruvate-dependent inhibition of histone deacetylases. Cancer Res. 66:11560-11564 (2006).

33. M. Ristow. Oxidative metabolism in cancer growth. Curr. Opin. Clin. Nutr. Metab. Care 9:339-345 (2006).

34. P. L. Pedersen. The cancer cell's "power plants" as promising therapeutic targets: an overview. J. Bioenerg. Biomembranes. 39:1-12 (2007).

35. S. Itagaki, E. Gopal, L. Zhuang, Y. J. Fei, S. Miyauchi, P. D. Prasad, and V. Ganapathy. Interaction of ibuprofen and other structurally related NSAIDs with the sodium-coupled monocarboxylate transporter SMCT1 (SLC5A8). Pharm. Res. 23:12091216 (2006). 